Conclusion Considering the nonspecific symptoms of the disease that greatly correlate with functional difficulties the number of negative findings is not surprising. But since gastroscopy is the most sensitive method of confirmation/ exclusion of the disease itself it is clear that a high number of negative findings point to the necessity of developing clearer guidelines to avoid unnecessary endoscopies. Furthermore, comparing our results with the results of similar foreign studies we can say that we are within the world average and that this indeed is a global problem that requires team effort especially at a time when the number of endoscopic procedures grows rapidly every day due to increased endoscopic possibilities.

\section{THE COINCIDENCE OF ROTOR AND GILBERT SYNDROME - A CASE REPORT}

Antonija Vuković* ${ }^{*}$ Vanda Žitko. KBC Split

10.1136/archdischild-2021-europaediatrics.283

Rotor syndrome is an autosomal recessive hereditary conjugated hyperbilirubinemia characterized by the appearance of mild, intermittent jaundice in otherwise asymptomatic patients. The prevalence of the disease is unknown but is assumed to be very low $(<1: 1000000)$. The diagnosis is made on the basis of elevated levels of conjugated bilirubin and elevated levels of coproporphyrin in the urine, at the expense of coproporphyrin I, with the exclusion of other liver diseases. We will present the case of a seventeen-year-old boy who was hospitalized in our Clinic in 2019. due to cholestatic jaundice that manifested after a short-term febrile illness treated with antibiotics. In 2015., we proved that the young man was heterozygous for Gilbert syndrome (familial benign unconjugated hyperbilirubinemia), but in the initial laboratory findings during this hospitalization we isolated total bilirubin $124 \mathrm{umol} / \mathrm{L}$ of which 104 umol/L was conjugated (83.8\%), which is not a characteristic of Gilbert syndrome. Clinically, he was in good general condition, had normocolored stool with dark orange urine (bilirubin in urine +++ ). There were no signs of hemolysis, alpha-1-antitrypsin as well as ceruloplasmin arrived at normal values, markers of hepatitis were negative, and synthetic and metabolic function of the liver was preserved. Antinuclear antibodies arrived borderline positive $(1: 80)$ but all of the other autoantibodies of connective tissue diseases were negative. Microbiological stool analysis (culture, parasite antigen detection) was negative. Abdominal ultrasound and MRCP did not show chnages in the morphology of the liver, cholecyst, biliary tree, and pancreatic duct. After all of the diagnostic procedures that excluded other liver diseases (infections, autoimmune diseases, pathomorphological changes in the liver and biliary tree), we assumed that it was another inherited disease of bilirubin metabolism, this time characterized by direct hyperbilirubinemia - Rotor or Dubin Johnson syndrome. To distinguish between the two syndromes, porphyrins were made in 24-hour urine. They were elevated at the expense of coproporphyrin I $(527.0 \mathrm{nmol} / \mathrm{dU}$; ref. Limit 7 $\mathrm{nmol} / \mathrm{dU}$ ) which supports the diagnosis of Rotor syndrome in a patient diagnosed with Gilbert's syndrome. Researching the available literature, we did not find the coincidences of
Gilbert and Rotor syndrome described so far, but this case warned us that regardless of the rare incidence of Rotor syndrome, it should always be considered in any patient who has chronic but mild hyperbilirubinemia at the expense of conjugated bilirubin.

\section{AN UNEXPECTED CAUSE OF VOMITING IN A SIX-YEAR- OLD GIRL}

Vlatka Konjik*, Nikolina Hamidović, Katarina Raković Amidžić, Ružica Ivelj, Dalibor Divković. University Hospital Centre Osijek, Department of Pediatrics

10.1136/archdischild-2021-europaediatrics.284

A six-year-old girl was admitted to Pediatric Intensive Care Unit in University Hospital Centre Osijek, Croatia, because of vomiting and severe hyponatremic dehydration, uremia and oliguria. Per anamnesis, her psychomotor development was normal. Except for having bronchitis, leading to one hospitalisation, she didn't have other serious illness or allergies.

The girl was admitted afer 4 days of vomiting, about 10 times in total. She didn't tolerate anything by mouth. Last stool was the day before her admission, normal. She was afebrile, sporadically ill. At the admission, she was conscious, afebrile, severely dehydrated, with sunken eyes, dry mouth and sticky tongue. She had no pain. From the initial laboratory: $\mathrm{Na}$ 113, Cl 62, BUN 35, creatinine 289, CRP 41, leukocytes 21; liver enzymes, serum amylase and lipase were normal. Abdominal ultrasound showed hepatic-like kidneys, with erased corticomedullary differentiation, as in acute kindey failure, and a lot of fecal mass in the intestines. Dehydration and hyponatremia were corrected, diuresis improved. Antibiotics were initiated because of elevated inflammatory markers. After the initial improvement, motility problems complicated her recovery. She vomited once daily, and had soft or loose stools. The abdomen was moderately distended. Control abdominal ultrasound showed liquid gastric content, now kidneys were normal, but no peristalsis. The abdominal X-ray showed one aeroliquid level, without distension. Rotavirus was isolated in the stool, which could explain gastroparesis and paralitic ileus. Nasogastric tube was placed, with gastroprotective agents and probiotics. In the following days, severe abdominal distension developed. Oral feeding was stopped, except of minimal feeding via enteral pump, but the intestinal motility did not improve. By then, she had no stool and via nasogastric tube green liquid content was evacuated daily. Nevertheless, her overall condition was stable, she remained afebrile and without pain. On the fourteenth day, endoscopy was performed. The gastroscope was inserted deep in the small intestine, there was liquid content visible aborally, but the site of the obstruction could not be visualised. Colonoscopy showed normal stool in the lumen. Abdominal MR was done, showing severe jejunal distension and transition zone toward ileum (which was normal in width) with suspected foreign body $27 \times 24 \times 38$ $\mathrm{mm}$ in size, and colapsed colon distally. Surgery was done, and the object that obstructed the lumen of the small intestine was removed - a piece of polyurethane foam. The polyurethane foam is used for filling and sealing in the construction. The girl did not remember swallowing that object, and after the surgery she recovered completely. 